Submission ID: 43952

\title{
Alternative Ways to Formalize Knowledge for Petrophysical Inversion
}

\section{B.N. Enikeev* (Pangea Inc.)}

\section{SUMMARY}

Robust petrophysical inversion in addition to logging data (matrix $Y$ ) requires additional information about the desired physical properties (matrix $X$ ) and their interrelationships. This approach allows us to regularize the solution and avoid petrophysical unreality. The author earlier proposed to classify alternatives for specifying such interrelationships of physical properties using cluster analysis and regression. The paper uses a sample of petrophysical data published in open press to compare cluster-type algorithms of mixture type and cluster regression and to recommend improvements. 


\title{
Альтернативные пути формализации знаний для петрофизической инверсии
}

\author{
Еникеев Б.Н. (АО "ПАНГЕЯ")
}

\section{Введение}

Устойчивая петрофизическая инверсия, помимо данных каротажа (матрица Y), требует привлечения дополнительной информации об искомых физических свойствах (матрица X) и их взаимосвязях. Такой подход позволяет регуляризировать решение и исключить его попадание в петрофизически нереальную область. Традиционно к дополнительным взаимосвязям [2] относят связи пористости и глинистости, пористости и содержания связанной воды,изменения содержания компонент породы с глубиной. Простейшим способом формализации такого подхода является использование гауссовского распределения для задания априорной информации об ожидаемых значениях матрицы искомых свойств пород. Вдохновляющая простота технологии использования такого приёма противоречит эмпирическим данным о характере распределения искомых свойств пород. Ранее [1-3] автором тезисов была предложена классификация альтернатив для задания таких взаимосвязей физических свойств (включая и петрофизические соотношения, и оконтуривающие данные измерений полигоны, и сети Kohonen). В докладываемой работе сравниваются подходы, основанные на кластерных алгоритмах типа смесей и на кластерной регрессии по выборке петрофизических данных, опубликованных в открытой печати.

\section{Используемые данные}

В открытой печати рядом авторов опубликованы таблицы данных, включающие измерения скорости упругих волн, пористости и проницаемости карбонатных горных пород. Нами использовалась 4 ре разные коллекции образцов, в частности, приведенные в [1-5]. Представление о характере этих взаимосвязей дает рис. 1.

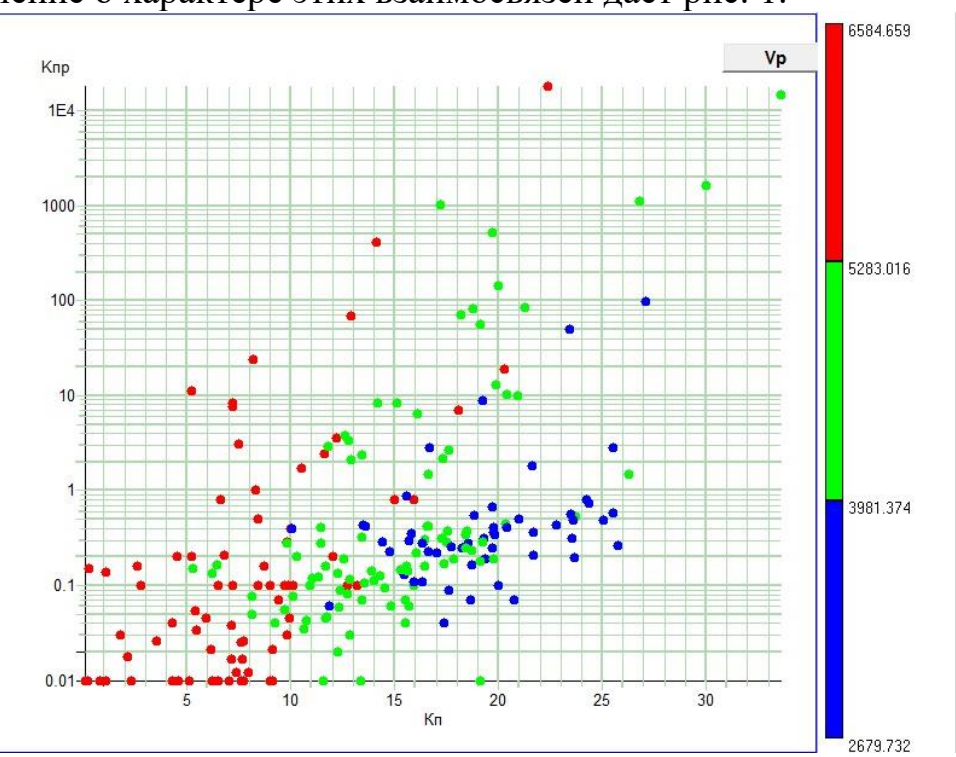

Рис 1 Корреляиионное поле пористость-проницаемость по данным теста (иветокод скорость подобных волн при давлении обжатия 40МПА).

Как видно из рисунка, плотность распределения соотношений связи пористости и логарифма проницаемости для используемых данных не является нормальной.

\section{Применяемые алгоритмы}

Для кластеризации использовались три алгоритма (кСредних, смеси многомерных нормальных распределений и размытых средних), а также алгоритм кластерной регрессии с прямой 


\section{EAGE}

оптимизацией методом Rosenbrock. Как видно из выбора состава алгоритмов, мы сочли необходимым учесть возможность выявления кластеров в виде вытянутых эллипсоидов, что мотивируется рисунком. Выбор в состав тестируемых алгоритмов расщепления смесей мотивировался также наличием у автора личного опыта работы с подобным алгоритмом и знания требуемых программных усовершенствований и модификаций. При проведении расчетов входные данные масштабировались по средним и дисперсиям. После получения результатов проводился обратный пересчет.

\section{Полученные результаты}

Результаты расчетов имеют цифровой вид, но более наглядна приводимая ниже их графическая иллюстрация (Рис 2-5).
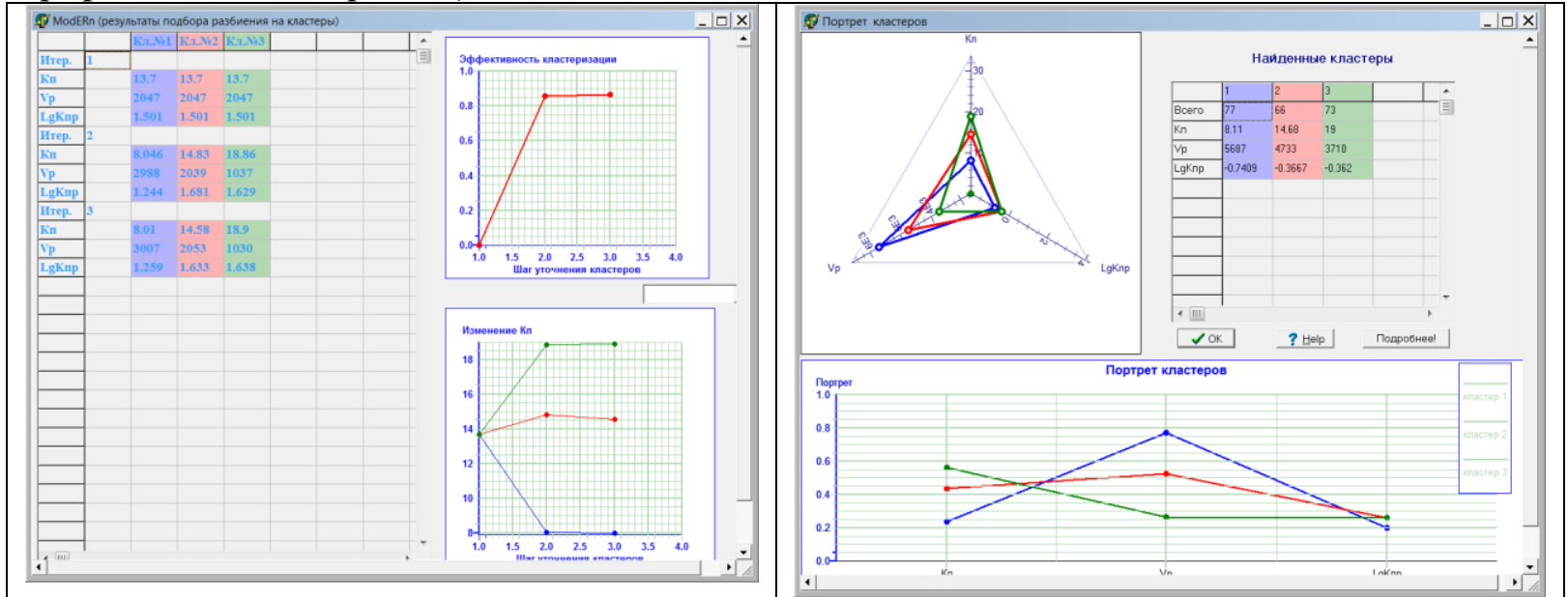

Рис 2 Результаты по kМеап (слева критерий выбора кластера справа - портрет кластеров)
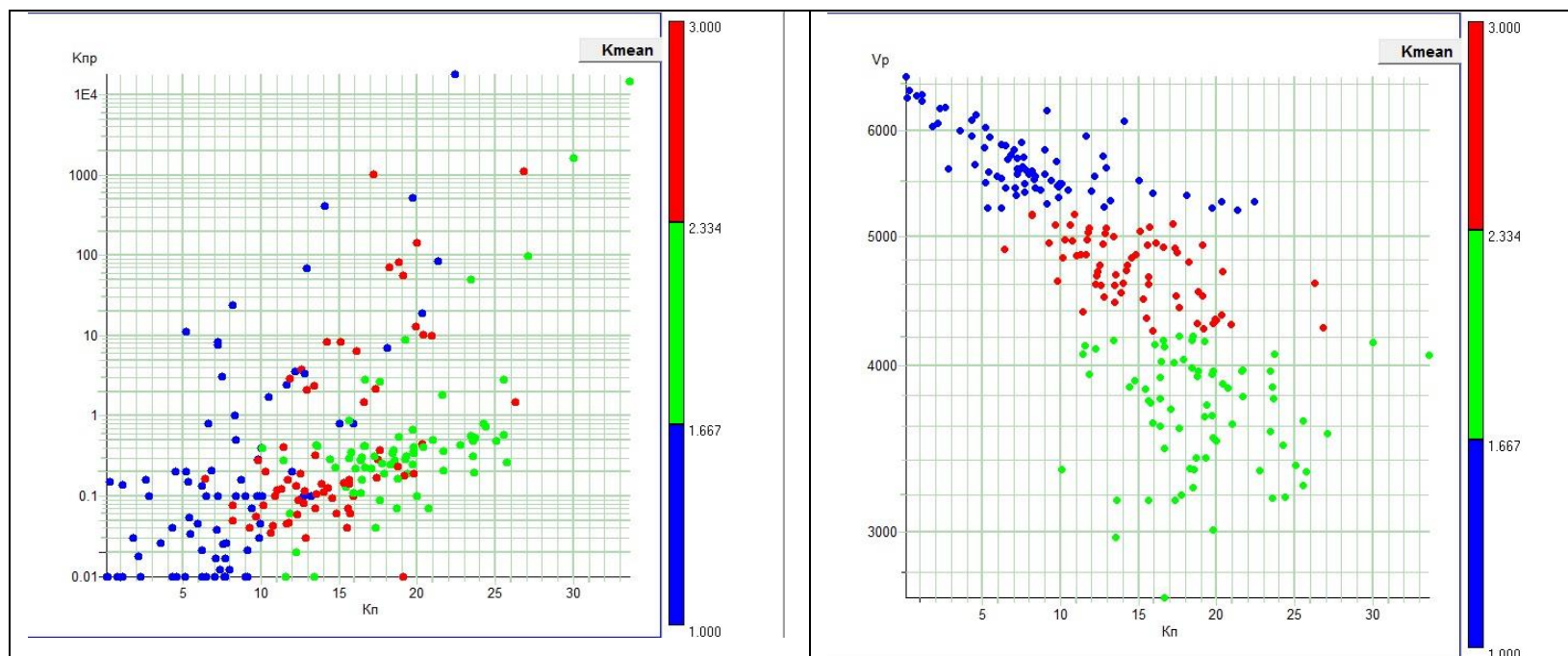

Рис 3 Результаты по kМеап (слева - критерий выбора кластера, справа - портрет кластеров). 

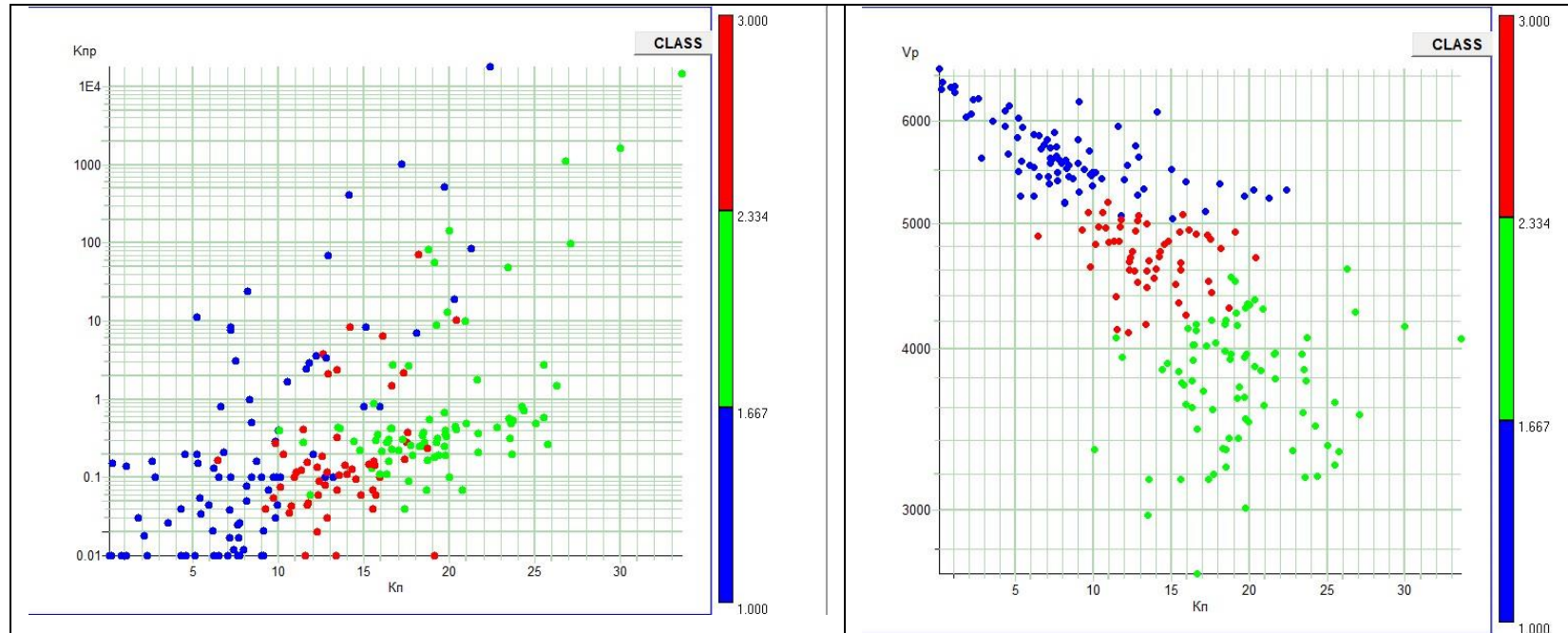

Рис 4 Окончательные результаты по FCM (слева - критерий выбора кластера справа портрет кластеров).

Алгоритм самообучения с помощью кластерной регрессии значимо зависит от начальных приближений и, поэтому, нуждается в доработке. Пример получаемого с его помощью результата приведен на Рис 5.

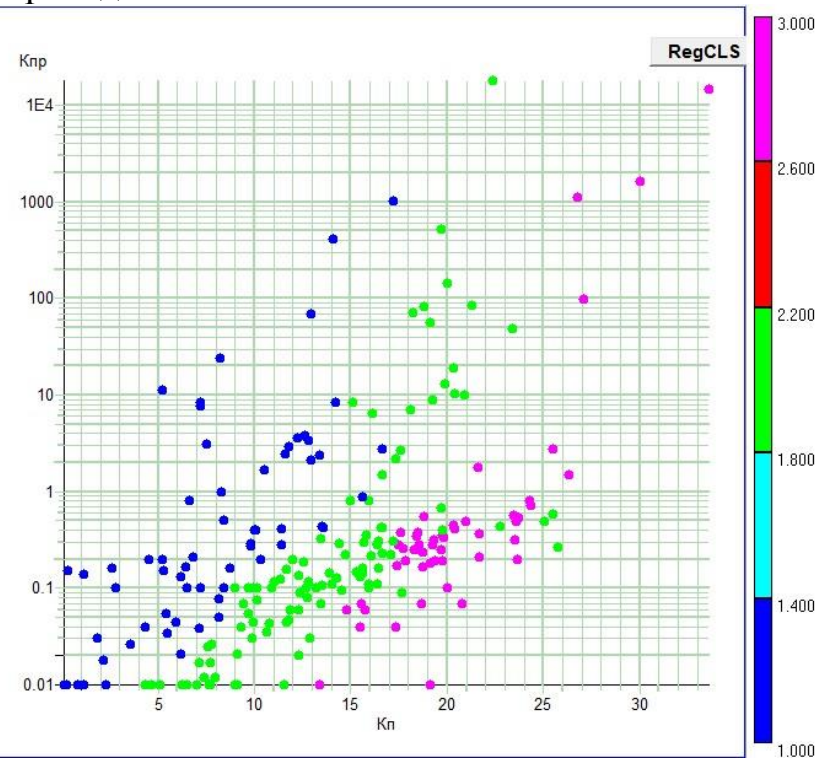

Рис 5 Результаты по алгоритму самообучения с помощью кластерной регрессии.

Сопоставление Рис 3 и Рис 4 показывает не только расщепление кросс-плота пористостьпроницаемость, но и большое сходство в данном случае результатов, получаемых по процедурам, реализующим указанные алгоритмы Такой вывод познавателен, но иллюстрирует, что при стандартном задании параметров процедур определяющим оказывается выделение сгустков, просматриваемых на кросс-плоте пористость - скорость продольных волн. Такой результат не показывает причин диффузии вниз точек взаимосвязи пористости со скоростью продольных волн. Вложить в петрофизически-необученный алгоритм подобную информацию может профессионал. Вместе с тем попытки выделить эту область на кросс-плоте с помощью полигона с дальнейшим переносом на кросс-плот пористость-проницаемость не дает желаемой дифференциации проницаемости на гидравлические единицы (что может быть объяснено одновременным влиянием и компонентного состава, и пороаспектного отношения). 


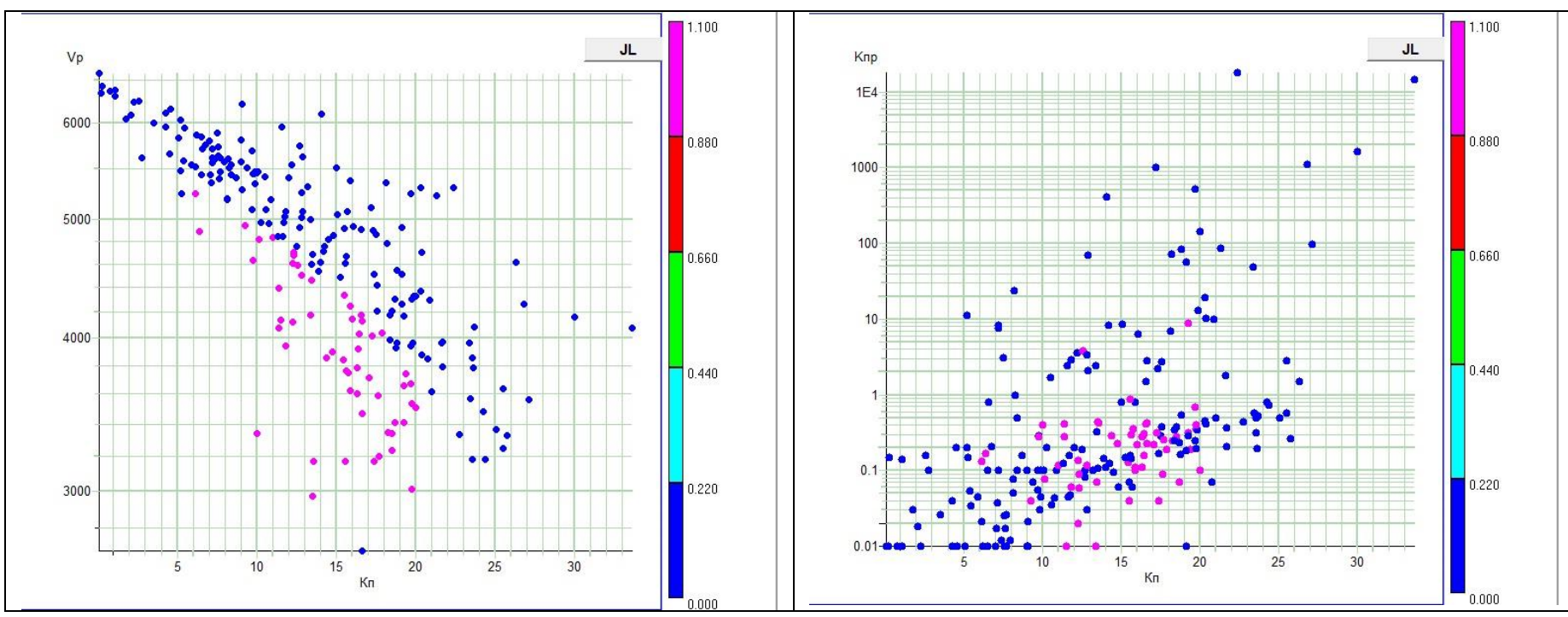

Рис 6 Корреляичонные поля, на которых выделена аномалия в зависимсти пористость скорость продольных волн.

Поиск компромисса в решении подобной недоопределенной задачи явно выходит за её рамки и требует расширения перечня измерений. В этой связи уместно напомнить, что наша цель была намного скромнее - изучение возможности аппроксимации областей сгущений искомых петрофизических переменных с применением различных алгоритмов. Алгоритмы, представляющие кластеры в виде многомерных эллипсоидов с оцененными средними и с ковариациями, эту задачу решают успешно. К сожалению, обилие настраиваемых параметров требует больших объёмов обучающей выборки. Альтернативы приведенному выше выбору алгоритмов, на взгляд автора, целесообразно искать в алгоритмах, менее требовательных к объёму обучающей выборки и более специализированных и чутких к априорной информации.

\section{Выводы}

1. При петрофизической инверсии целесообразно представлять ограничения на область изменения искомых переменных результатов в виде смесей многомерных гауссовских распределений.

2. Актуальной остается задача обучения алгоритмов общего вида специфике данных, для обобщения которых они применяются

\section{Литература}

1. Еникеев Б.Н. Изучение влияния эффективного давления на скорости упругих волн с целью отбора устойчивых взаимосвязей Geomodel 2016 - 18th Science and Applied Research Conference on Oil and Gas Geological Exploration and Development

2. Еникеев Б.Н. Эмпирическое сопоставление избранных взаимосвязей скорости продольных волн песчаников с их пористостью, глинистостью и давлением Туumen 2017

3. Элланский М.М., Еникеев Б.Н. Использование многомерных связей в нефтегазовой геологии. Недра, 1991, 207стр

4. A. Hairabian, F. Fournier, J. Borgomano and S. Nardon Sueto Quantitative DEPOSITIONAL FACIES, PORE TYPES ANDELASTIC PROPERTIES OF DEEP-WATER GRAVITY FLOW CARBONATES Journal of Petroleum Geology, Vol. 37(3), July 2014, pp 231-250

5. Soete J., Kleipoo L.M., Claes H. Acoustic properties in travertines and their relation to porosity and pore types Marine and Petroleum Geology 59 (2015) 320-335 


\section{References}

1. Enikeev B.N. The study of the effect of effective pressure on the velocity of elastic waves in order to select stable relationships Geomodel 2016 - 18th Science and Applied Research Conference on Oil and Gas Geological Exploration and Development

2. Enikeev BN An empirical comparison of selected relationships between the speed of longitudinal waves of sandstones and their porosity, clayiness and pressure Tyumen 2017

3. Ellansky M.M., Enikeev B.N. The use of multidimensional relationships in oil and gas geology. M .: Nedra 1991 208p.

4. Hairabian, F. Fournier, J. Borgomano and S. Nardon Sueto Quantitative DEPOSITIONAL FACIES, PORE TYPES ANDELASTIC PROPERTIES OF DEEP-WATER GRAVITY FLOW CARBONATES Journal of Petroleum Geology, Vol. 37(3), July 2014, pp 231-250

5. Soete J., Kleipoo L.M., Claes H. Acoustic properties in travertines and their relation to porosity and pore types Marine and Petroleum Geology 59 (2015) 320-335 